\title{
Wissens- und Technologietransfer in europäischer Dimension: Service Center für internationalen Wissens- und Technologietransfer (SeWiTec) der Technischen Fachhochschule Wildau
}

\author{
Sabine Gossner, Daniela Gorsler
}

\section{Einleitung}

Die Technische Fachhochschule Wildau zeichnet sich als eine forschungsaktive Fachhochschule aus, die engen Kontakt mit Praxispartnern pflegt. Die Aktivitäten im Wissens- und Technologietransfer erstrecken sich dabei auf alle Ebenen: Vorhaben mit Praxispartnern und anderen Wissens- und Forschungseinrichtungen werden in regionaler, nationaler und internationaler Zusammenarbeit entwickelt. Im Bundesdurchschnitt hält die TFH Wildau eine Vorreiterrolle bezüglich der eingeworbenen Drittmittel pro Professur inne (Anmerkung 1). Ziel ist, an dieser Position festzuhalten bzw. diese noch weiter auszubauen. Gerade im Hinblick auf den Aufbau von internationalen Netzwerken und der Einwerbung von Mitteln aus Programmen der Europäischen Union ist Potenzial vorhanden, das in den nächsten Jahren verstärkt werden soll. Diese Entwicklung ist an die Tatsache gebunden, dass auch nationale Fördermittel immer mehr an die Richtlinien europäischer Unterstützungsprogramme angepasst werden und somit auch nationale Projekte verstärkt internationale Komponenten aufweisen sollen. So erhalten z. B. Vorhaben im Programm ProInno II mit Partnern aus anderen EU-Ländern 10 \% mehr Förderzuschlag. Außerdem findet in den meisten regionalen als auch nationalen Programmen die EU-Definition für kleine und mittelständische Unternehmen Anwendung (Anmerkung 2). Bereits jetzt gewinnt die Durchführung von europäisch bzw. international orientierten Vorhaben an Relevanz, von der nicht nur die Hochschule profitiert. Auch für die wissenschaftliche Profilierung von Hochschulangehörigen ist die erfolgreiche Umsetzung europäischer Projekte ein Plus im Werdegang, das nicht zu unterschätzen ist.

\section{SeWiTec - Eine Piloteinrichtung im Land Brandenburg}

Die Technische Fachhochschule hat diesen Trend früh erkannt und eine Unterstützungsinstitution eingerichtet, das den Hochschulangehörigen bei der Entwicklung und Durchführung europäischer und internationaler Vorhaben und Projekte zur Verfügung steht: Seit Mai 2004 übernimmt das Service Center für internationalen Wissens- und Technologietransfer (SeWiTec) diese Aufgabe. Es handelt sich dabei um eine Piloteinrichtung im Land Brandenburg, und auch in anderen Bundesländern sind vergleichbare Einrichtungen an Fachhochschulen eher selten. Im Gegensatz zu den Universitäten, die in der Regel ein EU-Forschungsreferat führen, verfügen die meisten Fachhochschulen in Deutschland nicht über eine gesonderte Einrichtung für die internationale Komponente des Wissens- und Technologietransfers. Äquivalente Einrichtungen sind z. B. zu finden an der Hochschule Mannheim oder an der Pädagogischen Hochschule Ludwigsburg. Diese Einrichtungen stehen allerdings länderübergreifend zur Verfügung und decken die Belange mehrerer Hochschulen ab (Anmerkung 3). Ansonsten übernehmen die Transfereinrichtungen oder Forschungsreferenten an Fachhochschulen auch diese spezielle Beratungskomponente. Allerdings ist die Entwicklung von europäischen oder internationalen Vorhaben und die Akquise von Fördermitteln aus der Europäischen Union eine „Wissenschaft für sich“, die besonderes Augenmerk verlangt. Die ohnehin schon überlasteten Beratungsstellen an Hochschulen können diese Mehrleistung nur schwer aufbringen. Eine Entlastung für die Hochschulangehörigen ist damit nicht unbedingt gewährleistet und die anfängliche Motivation, sich europäisch zu orientieren, verpufft durch den erheblichen Aufwand, den europäische Projektvorhaben und die Antragsstellung mit sich bringen. Genau hier setzt nun die Service-Einrichtung an der TFH Wildau an: Das SeWiTec bietet umfassende Unterstützungen in allen Bereichen der Antragstellung und Projektarbeit und ist speziell auf die Bedürfnisse europäischer Projektzusammenarbeit ausgerichtet. Entsprechend der Aufgabe der TFH Wildau, als Motor für die Region zu dienen, stehen die Leistungen der Service-Einrichtung auch Unternehmen aus der Region offen.

\section{Aufgabenspektrum des SeWiTec}

Gemäß dem Motto „Von der Idee zum Projekt“ umfasst das Aufgabenspektrum des SeWiTec alle Aktivitäten der internationalen Projektentwicklung, -beantragung, und -umsetzung. Dies bezieht sich sowohl auf Vorhaben der angewandten Forschung und Entwicklung, auf den Aufbau von Studiengängen und berufsqualifizierenden Weiterbildungsangeboten sowie auf Maßnahmen der Hochschulentwicklung und des Hochschulmanagements. Ein zweiter Aufgabenbereich der Service-Einrichtung liegt im Aufbau bzw. in der Erweiterung transnationaler Vernetzungen zwischen der Technischen Fachhochschule Wildau und ausländischen Hochschulen, Forschungsinstitutionen und Unternehmen sowie die Integration der Hochschule in europäische und internationale Forschungsnetzwerke. Den dritten Tätigkeitsschwerpunkt des SeWiTec bildet die Information über Förderprogramme und die Öffentlichkeitsarbeit. 


\subsection{Beratung bei der Projektentwicklung und Antragstellung}

Die Beratungs- und Unterstützungsleistungen des SeWiTec bei der Entwicklung und Beantragung internationaler Projekte richtet sich nach der Rolle der Hochschule im Projekt - Koordinator, Konsortialpartner oder Auftragnehmer - und der Vorerfahrung der beteiligten Akteure. Das Leistungsspektrum reicht von der Erstberatung der Wissenschaftler und der Konkretisierung der Projektidee über die Fördermittelrecherche, den Kontaktaufbau zu Förderinstitutionen und die Anpassung des Projektdesigns an die inhaltlichen Prioritäten und Bewertungskriterien der Ausschreibungen bis hin zur zeitlichen Koordination der Antragstellung oder sogar der inhaltlichen und finanziellen Ausgestaltung des Antrags. Werden weitere Projektpartner benötigt, akquiriert das SeWiTec diese aus den breit gefächerten regionalen und internationalen Netzwerken der Hochschule. Da neben der Fachkompetenz die persönliche Komponente in transnationalen Projekten eine zentrale Rolle spielt, richtet das SeWiTec bei Bedarf Meetings zum Zusammenführen der Partner in Wildau aus oder bereist die Partnerinstitutionen im Ausland. Schließlich gewährleistet es eine fristgerechte und formal korrekte Einreichung der Anträge.

Die Arbeit des SeWiTec richtet sich vorrangig an den Fachdisziplinen der Technischen Fachhochschule Wildau aus: Luftfahrttechnik, Logistik, Nano- und Materialtechnologie, Biosystemtechnik, IKT und E-Learning sowie Wirtschafts- und Regionalwissenschaften. Es unterstützt auch Projektansätze in anderen Themenfeldern, wenn dort die Expertise der Hochschullehrer gefordert ist und die Projekte zur Steigerung der internationalen Kompetenz der Hochschule beitragen können.

\subsection{Unterstützung bei der Projektumsetzung}

Als das SeWiTec im Jahr 2004 seine Arbeit aufnahm, liefen an der Technischen Fachhochschule Wildau bereits mehrere EU-Projekte und internationale Aktivitäten. Für diese sowie inzwischen neu bewilligte Vorhaben bietet das SeWiTec eine umfassende Unterstützung beim administrativen, finanziellen und organisatorischen Projektmanagement. Konkret bedeutet dies die Mitwirkung am Berichtswesen, die Beratung in Vertragsfragen, die Vorbereitung von Kostenübersichten und von Auditprüfungen, die projektbezogene Öffentlichkeitsarbeit und Ergebnisverbreitung sowie die Organisation und Durchführung von Projekttreffen (siehe Abb. 1). Eine enge Zusammenarbeit mit dem jeweiligen Projektleiter und mit den beteiligten Institutionen innerhalb der Hochschule - z. B. die Hochschulleitung, das Sachgebiet Haushalt und das Akademische Auslandsamt - sichert die erfolgreiche Umsetzung der Projekte.

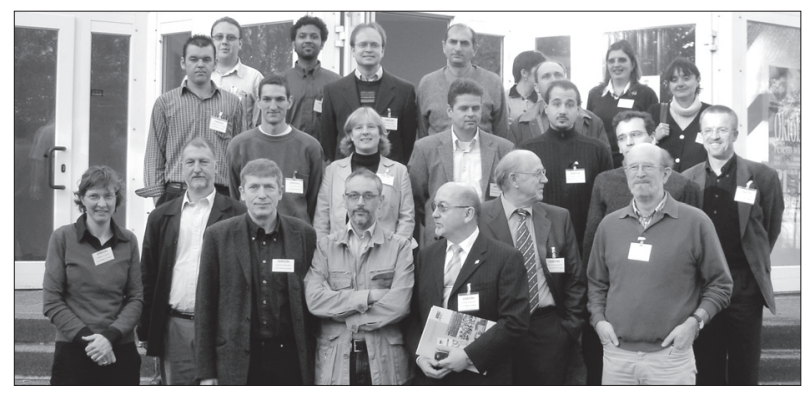

Abb. 1: Partnermeeting im ODEON-Projekt

\subsection{Ausbau der transnationalen Vernetzung}

Die Hochschulleitung der Technischen Fachhochschule Wildau hat die neuen Herausforderungen durch die Internationalisierung der Hochschullandschaft frühzeitig erkannt und arbeitet gemeinsam mit den einzelnen Hochschullehrern und dem SeWiTec intensiv am Aufbau und an der Festigung internationaler Netzwerke. Mittlerweile gehören Hochschulen, Unternehmen und öffentliche Einrichtungen in ganz Europa und in Drittstaaten wie der Ukraine zum Partnernetzwerk der Fachhochschule. Besonders intensiv wurden seit 2005 Kontakte zu neuen Partnern in Ungarn, Polen, Kroatien und Bulgarien aufgebaut. Diese mündeten in Projektanträge in den Programmen TEMPUS und INTERREG III B CADSES sowie Leonardo da Vinci II. Im letztgenannten Programm wurde ein Pilotprojekt zur Entwicklung eines E-Learning-Kurses für Logistiker bewilligt, in das sich die Technische Fachhochschule Wildau seit Oktober 2005 mit der Entwicklung von vier E-LearningModulen einbringt.

Im Luftfahrtbereich wurde die europäische Vernetzung der Hochschule durch ihren Beitritt zum European Aeronautics Science Network vertieft (siehe http: //www.easn.net/). Auf einem Netzwerktreffen im April 2005 in Brüssel konnten neue Verbindungen zu Hochschulen in Großbritannien, Frankreich und den Niederlanden aufgebaut und Verbindungen zu Hochschulpartnern in Ungarn, Spanien und der Slowakischen Republik vertieft werden.

Seit September 2005 wird die transnationale Vernetzung von Seiten des Landes Brandenburg zunehmend unterstützt. Ziel ist, die Position des Landes in der europäischen Forschungslandschaft zu stärken und die Beteiligung von Brandenburger Einrichtungen an europäisch geförderten Projekten zu erhöhen. Das SeWiTec beteiligt sich an diesem Prozess und ist auf einem Arbeitstreffen von Mitarbeitern aus Hochschul- und Forschungseinrichtungen in der Vertretung des Landes Brandenburg beim Bund sowie in Brüssel vertreten. Dadurch können für die Projektarbeit wichtige Verbindungen zu EU-Kommissionsbeamten aus Brandenburg aufgebaut und Kontakte zur Landesvertretung in Brüssel vertieft werden.

\subsection{Informationsverbreitung und Öffentlichkeitsarbeit}

Die Information über Entwicklungen in der europäischen und nationalen Förderpolitik, über potenzielle Fördermöglichkeiten, aktuelle Antragsfristen und -bedingungen aber auch über die Aktivitäten und Arbeitsergebnisse des SeWiTec haben einen hohen Stellenwert in der Arbeit der Service-Einrichtung.

Die Information und Öffentlichkeitsarbeit erfolgt über verschiedene Wege, die sich in den vergangenen zwei Jahren bewährt haben:

- Informationsveranstaltungen zu aktuellen Programmaufrufen, zukünftigen Entwicklungen in der europäischen Förderpolitik sowie zu spezifischen Programmen wie dem 6 . und 7. Forschungsrahmenprogramm der EU oder den Strukturfonds.

- Homepage mit Kurzdarstellung der Dienstleistungen, Übersicht über die internationalen Projekte, Hochschulkooperationen und Netzwerke der Technischen Fach- 
hochschule Wildau, Informationen zu Veranstaltungen des SeWiTec und Überblick über laufende Förderprogramme (siehe http://www.tfh-wildau.de/sewitec).

- Elektronischer Newsletter „SeWiTec AKTUELL“ mit Informationen über die laufende Arbeit der Einrichtung, internationale Aktivitäten der Hochschule, aktuelle Ausschreibungen und Hinweise für die internationale Projektarbeit. (siehe http://www.tfh-wildau.de/sewitec/ index_1621.htm).

- Ein Flyer in deutscher und englischer Sprache, Pressemitteilungen und Fernsehberichte zu einzelnen Aktivitäten aber auch die Beteiligung an internationalen Messen wie „Innovationen, Technologien, Maschinen“ in Poznań stellen sicher, dass unterschiedliche Zielgruppen aus Wissenschaft und Wirtschaft adäquat über die Ziele und Aktivitäten des SeWiTec informiert werden.

\section{Herausforderungen an den europäischen Technologietransfer}

Neben Forschung und Lehre zählt der transnationale Wissens- und Technologietransfer heute zu den größten Herausforderungen, denen Hochschulen gegenüberstehen. Dabei gilt es, Strukturen und Prozesse für eine dauerhafte Kooperation mit Partnern aus Wissenschaft, Wirtschaft und Verwaltung zu etablieren, um gemeinsam neue Produkte, Verfahren und Dienstleistungen zu entwickeln. Bei dieser win-win-Konstellation profitieren die Hochschulen von einer besseren Ausstattung und dem Transfer ihres Know-how, einem Imagegewinn und größerer Attraktivität gegenüber Studierenden und künftigen Mitarbeitern. Unternehmen können durch die Zusammenarbeit Fachwissen von Beschäftigten aktualisieren und deren Qualifizierung steigern sowie neue marktfähige Produkte generieren.

Zahlreiche EU-Programme in Bildung, Forschung und einzelnen Fachpolitiken unterstützen den Technologietransfer zwischen Partnern in unterschiedlichen EU-Staaten und über die Grenzen der Europäischen Union hinaus. Die Förderkonditionen variieren zwar im Einzelnen, aber es lassen sich einige Grundprinzipien für die transnationale Zusammenarbeit ausmachen, die auch das SeWiTec in seiner Arbeit berücksichtigt:

- Europäische Dimension der Partnerschaft: Formale Voraussetzung für die Förderung eines Projektes ist i.d.R., dass sich mindestens drei Partner aus drei EU-Mitgliedstaaten oder Assoziierten Staaten zusammenfinden. In der Realität zeigt sich jedoch, dass die Europäische Kommission Projekte mit erheblich größeren Partnerschaften bevorzugt. Daher ist ein stabiles, kompetentes und breit angelegtes Partnernetzwerk, wie es die Technische Fachhochschule Wildau pflegt, ein zentraler Erfolgsfaktor.

- Komplementärfinanzierung: Die Kofinanzierung der Europäischen Kommission für Projekte ist als Ergänzungsfinanzierung gedacht und überschreitet selten Werte von 75 \% der förderfähigen Gesamtkosten eines geplanten Projektes. Die Partner müssen daher einen gewissen Prozentsatz als Eigenanteil aufbringen, was gerade bei kleinen Unternehmen oder Partnern in
Nicht-EU-Staaten zu erheblichen Belastungen führt. Das SeWiTec achtet hier auf eine ausgewogene Budgetierung und die Realisierbarkeit der Kofinanzierung.

- Aufwand bei der Beantragung und Realisierung europäischer Projekte: Gegenüber nationalen Vorhaben sind EU-Projekte durch einen erhöhten administrativen und koordinatorischen Aufwand gekennzeichnet. Bereits die Recherche und Sichtung der Antragsunterlagen, der Umfang und die Sprachvariante des Antrags sowie die langen und mehrstufigen Auswahlverfahren stellen höchste Anforderungen an den Leiter und die Mitglieder eines europäischen Projektkonsortiums. Die gemeinsame Erarbeitung von hochwertigen Produkten und die Entwicklung von zufrieden stellenden und verwertbaren Ergebnissen sind ebenfalls eine Herausforderung. Hier steht das SeWiTec den Hochschullehrern, der Hochschulverwaltung sowie den Partnern der Technischen Fachhochschule Wildau in allen Fragen unterstützend zur Seite.

In der transnationalen Zusammenarbeit kommen weitere Faktoren zum Tragen, die ein höheres Gewicht einnehmen als im nationalen Kontext. Eine starke transnationale Partnerschaft auf der Basis von gemeinsamen Projekterfahrungen und gegenseitigem Vertrauen ist eine zentrale Grundlage für erfolgreiche Projekte. Dies erfordert intensive und kontinuierliche Aktivitäten beim Aufbau und bei der Pflege eines Partnernetzwerkes und ein Verständnis für die politische, wirtschaftliche und institutionelle Situation in den Partnerländern. Durch Umstände wie ein verzögertes Zusammenfinden von Partnern aufgrund von sprachlichen, kulturellen und fachlichen Unterschieden, durch die Ungewissheit über die Eignung eines Partners für ein Projekt und die Heterogenität der Qualifikationen der beteiligten Personen wird der Wissens- und Technologietransfer erschwert und so manches Projekt gerät bereits in der Frühphase an seine Grenzen. Aufgabe des SeWiTec ist es, diese Unwägbarkeiten und Risiken zu minimieren und machbare Lösungen für transnationale Kooperationen zu entwickeln.

\section{Bilanz}

Das SeWiTec besteht seit mittlerweile zwei Jahren. Das Profil der Tätigkeit hat sich dabei kontinuierlich weiterentwickelt und verändert. Nachdem die Infrastruktur an der TFH Wildau schnell etabliert war, konnte die ServiceEinrichtung die Arbeit aufnehmen. In der Anfangsphase stand die Information über europäische Fördermöglichkeiten, über EU-Aktionsprogramme und über die Umsetzung europäischer Projekte im Vordergrund. Es galt, die Hochschulangehörigen für europäische Vorhaben zu sensibilisieren und über die Anforderungen zur Antragstellung in EU-Programmen aufzuklären. Neben der Vorbereitung auf die Gestaltung europäisch orientierter Vorhaben lag 2004 der Schwerpunkt auf Erstberatungen. In diesen Beratungen wurden Projektideen im Hinblick auf europäische Fördermöglichkeiten geprüft und weiterentwickelt. Dabei wurden auch die internationalen Kontakte der jeweiligen Dozenten sondiert und Möglichkeiten für die europäische Partnersuche aufgezeigt. An die Erstberatungen schlossen 
sich 2005 zahlreiche Folgeberatungen an, in denen die Projektvorhaben entsprechend weiterentwickelt und bis zur Antragstellung optimiert werden konnten. Die steigende Anzahl der Beratungen mit externen Partnern zeigt, dass die Leistungen von Unternehmen verstärkt nachgefragt wird. In erster Linie wurden dabei kleine und mittelständische Unternehmen vom SeWiTec beraten.

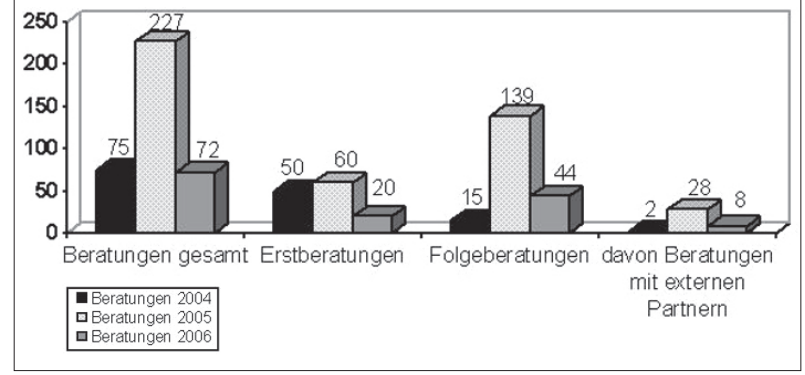

Abb. 2: Beratungen zur Projektentwicklung/Antragstellung des SeWiTec Mai 2004 bis April 2006.

Die Anzahl der begleiteten Anträge nahm kontinuierlich zu. Seit Mai 2004 hat das SeWiTec über siebzig Anträge begleitet (Zahlen beziehen sich auf den Zeitraum 2004-2005). Entsprechend der Vielfalt der Projektideen wurden Anträge in verschiedenen Förderprogrammen eingereicht. Die beantragten Vorhaben umfassten reine Forschungsvorhaben im Bereich Nanotechnologien oder Life Sciences, Entwicklung von Weiterbildungsangeboten oder E-Learning-Angeboten als auch Projekte in der Hochschulentwicklung. Folgende Unterteilung lässt sich vornehmen:

- Aktionsprogramme der Europäischen Kommission.

Dazu zählen u.a. das Forschungsrahmenprogramm der Europäischen Union, die EU-Bildungsprogramme wie Sokrates/Erasmus, Leonardo da Vinci, Minerva, Tempus und die Gemeinschaftsinitiativen INTERREG oder EQUAL.

- Strukturfondsmittel der Europäischen Union.

Das Land Brandenburg erhält als Ziel-1-Gebiet zur Stärkung der Wettbewerbsfähigkeit als auch zum Infrastrukturausbau Fördermittel aus dem Europäischen Fonds für regionale Entwicklung (EFRE) und zur Schaffung von Arbeitsplätzen und zu Qualifizierung von Beschäftigten Fördermittel aus dem Europäischen Sozialfonds (ESF) (Anmerkung 4).

- Nationale Förderprogramme der Bundesministerien, die teilweise mit internationalen Partnern umgesetzt werden können.

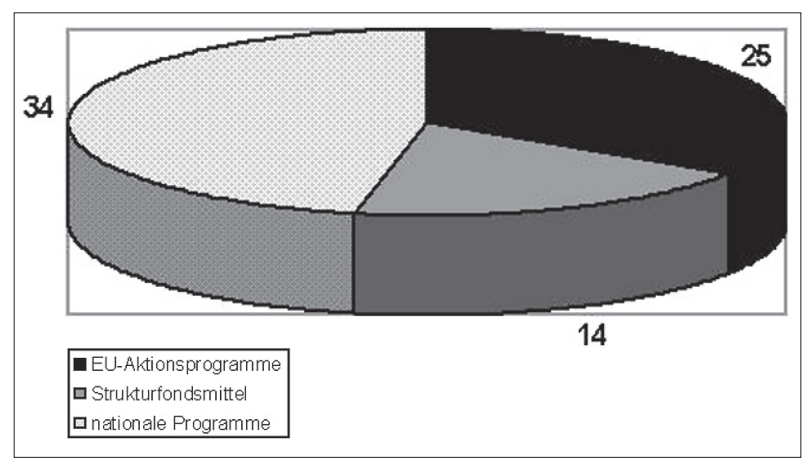

Abb. 3: Antragstellung nach Förderprogramm.
Es versteht sich von selbst, dass die Zahl der eingereichten Anträge gegenüber der der bewilligten Anträge überwiegt. Gerade bei den EU-Programmen ist mit einer langen Evaluierungsphase zu rechnen. Wartezeiten von bis $\mathrm{zu}$ sechs Monaten sind eher die Regel als die Ausnahme. Die Früchte einer erfolgreichen Antragstellung können erst spät geerntet werden. Bisher sind seit Mai 2004 elf positive Bewilligungsbescheide für Vorhaben erteilt worden, die durch das SeWiTec betreut wurden. Insgesamt betreut die Service-Einrichtung derzeit 22 EU-Projekte bzw. Vorhaben, die mit Mitteln von der Europäischen Union finanziert wurden, und bei denen die TFH Wildau als Koordinator oder Partner eingebunden ist.

\section{Fazit}

Entsprechend der Zielvorgaben der Service-Einrichtung konnte bisher die internationale und europäische Projektaktivität der TFH Wildau erheblich gesteigert werden. Durch die Beratungs- und Unterstützungsleistung des SeWiTec wird die Bereitschaft der Hochschulangehörigen gestärkt, ihre Vorhaben international auszurichten und dafür europäische Gelder zu beantragen. Die Hemmschwelle, EU-Gelder zu akquirieren und sich durch den „Förderdschungel Brüssel“ zu kämpfen, wurde weitestgehend abgebaut. Information und individuelle Beratung durch das SeWiTec haben diese Entwicklung maßgeblich beeinflusst. Außerdem tragen positive Erfahrungsberichte über EU-Projekte oder bewilligte Vorhaben dazu bei, die Motivation für EU-Anträge innerhalb der Fachhochschule zu steigern. Gleichzeitig unterstützt das SeWiTec die europäische Vernetzung und internationale Ausrichtung der TFH Wildau und erleichtert auf diese Weise die Kontaktaufnahme mit europäischen und internationalen Partnern.

Trotzdem kann nicht beschönigt werden, dass der Kampf um EU-Gelder hart ist, die Konkurrenz entsprechend groß. Die Anforderungen sind sehr hoch: Anträge mit gut aufgestellten Konsortien und überzeugenden, innovativen Projektvorhaben müssen qualitativ anspruchsvoll gestaltet sein, um das Gutachterverfahren positiv zu durchlaufen und die benötigte Punktzahl für eine Förderung zu erreichen. Auf diesen Prozess kann relativ wenig Einfluss genommen werden. Dennoch kann die Auswertung von Gutachterergebnissen von abgelehnten Anträgen hilfreich sein für eine weitere Antragsstellung. Das SeWiTec nimmt hier die entsprechende Optimierung von abgelehnten Anträgen vor.

Die Ausgestaltung der EU-Förderpolitik ab 2007 steht im Fokus der zukünftigen Arbeit der Service-Einrichtung. Verschiedene Förderprogramme werden neu definiert bzw. erhalten eine andere Ausrichtung. Dazu zählen u.a. das Forschungsrahmenprogramm der Europäischen Union sowie die EU-Bildungsprogramme, die beide im Mittelpunkt von Antragstellungen durch die TFH Wildau stehen. Aufgrund der langwierigen Verhandlungen über den Finanzhaushalt der Europäischen Union ist es wahrscheinlich, dass kein nahtloser Übergang zwischen der alten und neuen Förderperiode stattfindet und $u$. $U$. eine Förderlücke entstehen wird. Das SeWiTec beobachtet diesen Entwicklungsprozess intensiv, um früh genug 
förderrelevante Informationen an die Hochschulangehörigen weitergeben zu können.

Die intensive Beratungs- und Informationstätigkeit des SeWiTec wird beständig fortgesetzt, um Projektvorhaben im Entwicklungsprozess zu begleiten und zur Antragstellung zu optimieren. Darüber hinaus wird die Zusammenarbeit mit regionalen Unternehmen weiter vorangetrieben. Insgesamt stärkt die Arbeit der Service-Einrichtung die Stellung der Technischen Fachhochschule Wildau als drittmittelaktive Hochschule und festigt durch Vernetzungsaktivitäten mit europäischen und internationalen Kooperationspartnern deren Position im europäischen Raum.

\section{Anmerkungen}

1 Forschungslandkarte Fachhochschulen, Potenzialstudie, hrsg. vom Bundesministerium für Bildung und Forschung, Berlin 2004.

2 Empfehlung der EU-Kommission zur KMU-Definition 2003/ 361/EG in: Amtsblatt der Europäischen Union L 124 vom 25.02.2003, S. 36ff.

3 Fachhochschule Mannheim: http://www.koord.hs-mannheim.de/ EU-Forschungsreferat der Pädagogischen Hochschulen Baden-Württembergs: http://193.196.151.129/eu_forsch/

4 Operationelles Programm Brandenburg, Förderperiode 2000 bis 2006, Entscheidung C (2000) 43000 vom 29.12.2000, Landesregierung Brandenburg.

\section{Autoren}

Sabine Gossner M.A.

Technische Fachhochschule Wildau Service Center für internationalen Wissens- und Technologietransfer (SeWiTec)

Tel. +49 3375 508-673

sabine.gossner@tfh-wildau.de

Dipl.-Ing. Daniela Gorsler

Technische Fachhochschule Wildau

Branchentransferstelle Logistik (BTL)

Tel. +49 3375 508-276

daniela.gorsler@tfh-wildau.de 